\title{
Dynamo Catalogue: Geometrical tools and data management for particle picking in subtomogram averaging of cryo-electron tomograms.
}

\section{DANIEL CASTAÑO-DÍEZ ${ }^{1,2 *}$, MIKHAIL KUDRYASHEV ${ }^{2,3,4}$ AND HENNING $\underline{\text { STAHLBERG }}^{2}$}

1 BioEM Lab at C-CINA, Biozentrum, University of Basel, Mattenstrasse 26, CH4058 Basel, Switzerland

2 Center for Cellular Imaging and NanoAnalytics (C-CINA), Biozentrum, University of Basel, Mattenstrasse 26, CH-4058 Basel, Switzerland

3 Max Planck Institute for Biophysics, Max-von-Laue-Straße 3, 60438 Frankfurt am Main, Germany

4 Buchman Institute for Molecular Life Sciences, Goethe University of Frankfurt, Max-von-Laue-Straße 15, 60438 Frankfurt am Main, Germany

* Corresponding author:

Daniel Castaño-Díez

BioEM lab

C-CINA, Biozentrum, University of Basel

Mattenstrasse 26

4058 Basel

Switzerland

Email: daniel.castano@unibas.ch 


\begin{abstract}
Cryo electron tomography allows macromolecular complexes within vitrified, intact, thin cells or sections thereof to be visualized, and structural analysis to be performed in situ by averaging over multiple copies of the same molecules. Image processing for subtomogram averaging is specific and cumbersome, due to the large amount of data and its three dimensional nature and anisotropic resolution. Here, we streamline data processing for subtomogram averaging by introducing an archiving system, Dynamo Catalogue. This system manages tomographic data from multiple tomograms and allows visual feedback during all processing steps, including particle picking, extraction, alignment and classification. The file structure of a processing project file structure includes logfiles of performed operations, and can be backed up and shared between users. Command line commands, database queries and a set of GUIs give the user versatile control over the process. Here, we introduce a set of geometric tools that streamline particle picking from simple (filaments, spheres, tubes, vesicles) and complex geometries (arbitrary 2D surfaces, rare instances on proteins with geometric restrictions, and 2D and 3D crystals). Advanced functionality, such as manual alignment and subboxing, is useful when initial templates are generated for alignment and for project customization. Dynamo Catalogue is part of the open source package Dynamo and includes tools to ensure format compatibility with the subtomogram averaging functionalities of other packages, such as Jsubtomo, PyTom, PEET, EMAN2, XMIPP and Relion.
\end{abstract}

\title{
Keywords
}

Cryo electron tomography; subtomogram averaging; data management

\section{Abbreviations}

two dimensions / dimensional

3D three dimensions / dimensional

EM electron microscope

cryo-EM cryo-electron microscopy 
cryo-ET cryo-electron tomography

EMDB electron microscopy data bank (www.emdatabank.org)

FSC Fourier shell correlation

SNR signal-to-noise ratio

STA subtomogram averaging 


\section{Introduction}

\section{Subtomogram averaging from cryo-electron tomograms}

Cryo-electron tomography (cryo-ET), with its unique capacity for the three-dimensional (3D) visualization of macromolecular complexes in a close-to-native state, is a rapidly developing technology (Harapin et al., 2013; Lucic et al., 2013). Subtomogram averaging (STA) recovers the structure of a given macromolecule by locating multiple noisy copies of the object of interest in one or several tomograms, and integrating them into one or multiple structures with a higher signal-to-noise ratio (SNR) (Briggs, 2013). This integration typically consists of an alignment step that accurately locates and imparts a common orientation to the initially differently oriented particles, followed by averaging of the aligned particles and classification of the particles into more homogeneous groups. The procedure has been widely used, with applications ranging from large macromolecular complexes inside the native context (Beck et al., 2007; Kudryashev et al., 2013; Pigino et al., 2011) to membrane proteins on membranes (Faini et al., 2013; Pfeffer et al., 2012) or isolated protein complexes (Dudkina et al., 2011). Currently, 11\% of the structures deposited in the electron microscopy data bank (EMDB) were solved by STA. Generally, there is a correlation between the number of particles and the resolution obtained (Kudryashev et al., 2012), suggesting that a larger amount of processed particles results in a higher final resolution. Several structures determined by STA have sub-nanometer resolution, revealing the secondary structure of the proteins present (Bartesaghi et al., 2012; Schur et al., 2013; Schur et al., 2015); in each case, a large number of 3D subtomograms was required.

Several software packages are available for the 3D processing of multiple subtomograms. These include $A V 3$ (Forster et al., 2005), Jsubtomo (Huiskonen et al., 2010), PyTom (Hrabe et al., 2012), EMAN2 (Galaz-Montoya et al., 2015), PEET (Nicastro et al., 2006), Relion (Bharat et al., 2015), and Dynamo (Castano-Diez et al., 2012).

\section{Particle picking}

Subtomogram analysis starts with the extraction of subtomograms from one or several tomographic reconstructions. These $3 \mathrm{D}$ subtomograms are referred to as particles in the following. The location, orientation and exact number of particles of interest in tomograms are not defined a priori. In STA, "particle picking" commonly refers to a set of actions 
carried out automatically or with input from the operator, with the goal of approximately determining the positions and possibly also the orientations of subtomograms, to produce a set of particles, each particle containing a unique single copy of a macromolecule of interest, accompanied by adequately formatted metadata. Compared to two-dimensional (2D) particle picking in micrographs for single particle analysis, picking 3D particles from cryo-ET volumes has two specific difficulties. First, the data are 3D volumes which usually suffer from anisotropic resolution, making their visualization more complex. Second, particle identification is more challenging for both automated methods and human operators, since tomography typically images 3D particles in their native context as opposed to isolated single particles.

Further, in spite of the common goal, in tomography the generic term "particle picking" expresses different procedural approaches in different scenarios. Determination of particle locations might involve purely automatic methods relying on image analysis of the tomograms, such as a pattern matching-based identification, as implemented in molmatch (Frangakis et al., 2002), regular picking from a surface, e.g., a bacterial membrane (Amat et al., 2010), or picking from a tubular crystal (Bharat et al., 2012). Alternatively, particle picking might be based on visual inspection and manually targeting the structure of interest on a computer monitor. Integration of a priori information arising from geometric constraints is a frequent requirement: the particles to average might lie on cellular membranes (Kudryashev et al., 2013), on virus capsids (Huiskonen et al., 2010), on lipid vesicles (Faini et al., 2013), along the axial path of tubular structures (Nicastro et al., 2006; Pigino et al., 2011), or might be the repeating subunits that generate such structures. Sometimes, the particle coordinates might be additionally constrained by the presence of crystalline order (2D or $3 \mathrm{D})$ or symmetry, like the single vertices of an icosahedral virus (Gil-Carton et al., 2015), and the symmetry assumption might need to be partially weakened to accommodate the actual behavior of the observed data (Peralta et al., 2013). Also, related geometric surfaces might be fully or partially populated with particles (Maurer et al., 2013). The various possible particle arrangements require the use of different combinations of automated and manual particle picking procedures

Here, we present an extension to the Dynamo package, called Dynamo Catalogue, which facilitates automated or semi-automated subtomogram particle picking from various geometries used in STA, such as isolated particles, filaments, vesicles, arbitrary 2D surfaces and 2D and 3D crystals. This toolbox is an integral part of the Dynamo workflow and 
allows scripting of repetitive tasks through the command line. Dynamo Catalogue features a graphical user interface (GUI)-enabled toolbox that assists in the management of the data and metadata, including geometry and metadata conversions, and offers pipelines for the STA workflow from particle picking to STA and classification. Dynamo Catalogue is available within the Dynamo package as open source at http://www.dynamo-em.org.

\section{RESULTS AND DISCUSSION}

\section{Data management system in Dynamo}

In order to streamline STA projects we have implemented a database that integrates particle picking and extraction into the Dynamo workflow. The database is organized as a catalogue system with a user-accessible GUI (dynamo_catalogue_manager; here and below the Dynamo commands are stylized in courier font) and a set of command-line tools for scripting. The objects of the database are (1) links to tomograms and (2) models describing particle geometry (Figure 1). Properties of the tomograms include a link to the file location, defocus, magnification, and to the orientation of the missing wedge. Dynamo catalogues can be generated and administered using the GUI or a command line. They provide the following functionalities:

1. Tomogram browsing. Catalogues generate a gallery of thumbnails providing a quick overview of the data. GUIs for 3D tomogram viewing include tools for navigation of volumes designed for 3D particle picking (dynamo_slicer, dynamo_tomoview). Dynamo_preview allows large volumes to be viewed without loading them fully into memory. In the other viewers, a region of interest can be specified (in dynamo_preview), archived, loaded into memory, and fully annotated.

2. Geometric modeling. The user can choose a model describing the geometry of the particle distribution, i.e., depending on whether they are isolated particles, filaments, vesicles, etc. An extensive library of geometric models is available, adapted to the typical data collection geometries encountered in life-science specimens (Table 1). Each type of model has a set of methods that lead from the clicked model points to the final coordinates and initial orientations of particles. One or several models may be linked and stored for each tomogram.

3. Particle cropping. The catalogue stores the locations and orientations associated with the particles extracted from each tomogram. Upon cropping, the extracted particles are 
stored on the hard drive together with a Dynamo table. This table contains: the particle ID, the particle coordinates in the tomogram, the shifts and rotations required to align the particle to the average, the orientation of the missing wedge (details of the table format are available with the command dthelp). The Dynamo table can be used to start an STA project. Input/output in other formats (e.g., Relion .star) is also supported.

4. Each raw tomogram, 'cropping volume', from which the particle extraction is performed, is associated with down-sampled or filtered versions tuned for optimal display. This enables particle picking on up- or down-sampled or filtered volumes, and/or extraction from non-binned, non-filtered tomograms. Additionally, particle picking can be done on tomograms that have higher contrast at low resolution (e.g., volumes reconstructed via ART, SIRT, or SART (Kunz and Frangakis, 2014)), and afterwards particles can be extracted at the same locations from corresponding tomograms generated by weighted back-projection.

Along with these core functionalities, the catalogue provides further tools for the control of sets of tomograms:

- Estimations of the missing wedge present in each tomogram and comparison with nominal parameters provided by the user.

- Extensive documentation and step-by-step tutorials with simulated datasets. Dynamo functions and methods are documented in a cross-linked documentation system that is generally accessible via the help command of Matlab, as well as via the Dynamo help center GUI dhelp, or online through the dwiki command.

\section{Particle picking in 3D}

\section{Models}

Models are basic geometric objects, designed to facilitate particle picking provided and archived by Dynamo Catalogue. Models are linked to tomograms and fulfill two main roles: to annotate tomograms, and guide particle cropping (Figure 1). Internally, models are instances of a class of Dynamo model objects, defined in the Matlab language, and as such are accessible also from outside of the Dynamo framework, i.e., using the command line of Matlab, and available for user customized scripting applications. Since Dynamo can also be run using the freely-available Matlab runtime libraries, the models and all commands are 
also accessible from the standalone Dynamo console for users without a Matlab license.

While the structure of the models serves as a building unit for database organization and is common for each geometry type in the library, the set of available annotation tools varies for each model type (Table 1). In this respect, each model type can be seen as a particular way of transforming the sets of clicked points into sets of particle locations and orientations ("table points"). In the following, we describe the basic library of models and the tools included to support each specific particle picking geometry. A detailed hands-on description on how the user interacts with the different models can be found in the webpage https://wiki.dynamo.biozentrum.unibas.ch/w/index.php/Video_tutorials\#Star ting_Guide

\section{Isolated particles (model type general)}

In the simplest model, 3D locations determined on the screen are considered to be the particle positions. For the frequent cases where the intuitive approach of browsing slices up and down inside the tomogram to locate approximate centers proves impractical, the model provides access to other viewing options. One is concurrent viewing (Figure 2): the user depicts a Z-projection of the tomogram in the main image of the dynamo_tomoview browser. This allows the XY coordinates of single particles to be easily distinguished at the cost of loosing the Z-information. A click on a particle on this Z-projection will update a second image in the browser to show the $\mathrm{XZ}$ and $\mathrm{YZ}$ slices around the clicked position, giving the user the option to determine the Z-position with a second click. An additional alternative, appropriate for densely packed cellular contents, is viewing through local montages. Clicking on a point in a 3D slice will update an auxiliary image to show several slices of the neighborhood of the clicked position simultaneously. A second click on the montage can then be used to determine the particle position more accurately. Auxiliary images for concurrent and montage viewing can be tuned with their own visualization settings (such as contrast, bandpass, number of projected slices).

\section{Filaments (model type filament)}

In the basic functionality of the filament class, the user provides points lying on a "backbone" of a straight/bent filament, providing an axis that traverses the filament-like structure longitudinally. The location of the backbone can be picked through sequential inspection of Z-slices or refined on the slices orthogonal to the direction of the filament (Figure 3A, B). Positions of particles are computed based on further design decisions input 
by the user, who can aim at a distribution of particles located directly along the backbone of the filament or distributed (helically or otherwise) at a given distance from the backbone. The orientations of the particles are defined correspondingly.

\section{Arbitrary membranes (type membrane)}

This model type is used to pick particles embedded in closed or open surfaces of an arbitrary shape (Figure 4). The user clicks on points of the surface visible in 2D slices. Dynamo then computes a triangulation on the clicked points to generate a 3D "depiction mesh". This triangulation provides a smooth supporting surface to represent the membrane itself, and is used to define sets of particles for picking on a regular grid along the surface, the normal vectors being stored as initial orientations. These orientations are stored as a triplet of Euler angles, where only two angles are used to represent perpendicularity to the surface. The third angle, that represents rotation around the normal direction, has an arbitrary value. Thus, if the collected angular orientations are used to generate an initial template, this will appear as a rotational average around an axis normal to the membrane. This is however enough to start a refinement project, provided the alignment procedure includes a complete scan for this angle.

The triangulation is computed by application of the Crust algorithm (Amenta et al., 1998), which lets the user choose between open or closed surfaces. A manually defined, sparse, initial triangulation (Figure 4 D) can be smoothed by applying a subdivision scheme; selecting the option "create table points" in the model editor window allows the user to define the separation distance between the final particle positions which is then used to convert the manually clicked mesh into a smoothed, finer set of table points. It is often helpful to oversample the surface during particle picking and identify true particles later during subtomogram alignment based on their inter-particle distance (option "separation_in_tomogram" when designing an alignment project with the GUI dcp).

The membrane model also allows the user to fit ellipsoid membrane shapes. Biological membranes of nearly spherical or tubular objects such as the outer membranes of bacteria can be manually identified as a closed contour in electron tomograms, when displayed in the direction of the electron beam. However, when displayed in the direction of the tilt axis, the missing wedge effect in tilt-limited electron tomography renders horizontal membrane features untraceable and the displayed cross section of the membrane then looks like a pair of opposing half-moons. Consequently, the user can only click on parts of the membrane 
seen in side views of the particles. In such cases, the user has the option of computing an elliptical fit to all points selected on the visible part of the membrane in the slice and use this information to pick "top views" of the membrane proteins from this and other slices.

\section{Vesicles (type vesicle)}

This model type is useful for the extraction of membrane proteins embedded in the surface of lipid vesicles. It allows the user to identify the center of such vesicles and adjust the radius and mean expected particle distance on the surface. Alternatively, a user can manually click on the membrane of each vesicle in several Z-sections and ask the program to fit a sphere to the clicked points. In both cases, the vesicle geometry is transformed into a set of points with coordinates uniformly distributed on the vesicle surface, each point having an associated orientation representing the normal vector to the vesicle surface at the corresponding point. Further alignment and averaging can be performed by local angular refinement.

\section{Combined models}

Specific options allow orientations to be assigned to isolated particles based on their positions relative to other available models representing filaments, surfaces, or membranes. The example depicted in Figure 5 illustrates the combination of the general and filament models. In this case, dynein particles are distributed non-uniformly along microtubules. While each individual dynein particle has to be manually picked to obtain its coordinates, an estimate of their orientations is determined from their positions relative to the filament. Thus, relating the isolated particle model to a filament model provides an efficient way to extract a set of dynein particles with rough orientations for the generation of an initial average and further local refinement.

\section{Semi-automation tools}

Dynamo Catalogue allows the use of semi-automated particle-picking tools. These are integrated in the GUI interfaces for particle picking as optional supporting tools. The results delivered in real time can be immediately inspected and corrected, accepted or rejected, or used for iterative fine-tuning of automation parameters. The example in Figure 6 shows the automatic location of vesicular geometries through template matching using previously clicked coordinates as seeds. 


\section{Memory control}

Visualization of large volumes implies special requirements for memory management. Data sets reside on the hard disk, which may be in a remote location. Data set manipulation requires a smooth data flow between the hard drive and the computer RAM memory, which typically is limiting the accessible volume size. Ideally, a full tomogram should be loaded into the display computer's memory for rapid visualization. Filtering operations, projections through multiple slices to increase the displayed SNR, or scene rendering from different viewing angles, are all tasks that benefit from the availability of data in memory. However, loading full tomograms is often not feasible: For example, a tomogram of $4096 \times 4096 \times 2000$ voxels stored as 32-bit floating point values corresponds to a dataset of $\sim 128 \mathrm{~GB}$, which is beyond what current computers can comfortably display. A possible solution is chopping tomogram files into several files that can individually fit into the memory of the computer. These smaller files are then processed independently, possibly demanding a special treatment to account for the boundaries. Alternatively, the particles can be localized in down-sampled and filtered volumes, and then extracted from the nonbinned, original tomograms.

Dynamo Catalogue gives the user control of the memory trade-off. Volumes can be navigated offline, i.e., without preloading any data into memory, but accessing the volume on the hard drive (in dynamo_preview). Such off-line navigation can be used to choose regions of interest in the tomograms that are then read from the disk into memory, while possibly loading a down-sampled version of the tomogram to further reduce memory.

The memory control tools are embedded into the programs for volume visualization and connected to the system for automated archiving of information. The Catalogue stores the coordinates of the regions visited inside the indexed tomogram files, allowing the user to quickly continue or inspect work from a previous session or marked by a different user.

Identified areas of interest (e.g., a filamentous structure in a tomogram) can be registered and cropped for processing online.

\section{Geometric manipulations on subtomograms}

In addition to the tools for tomogram archiving and annotation, Dynamo Catalogue includes further tools to manipulate $3 \mathrm{D}$ data at the subtomogram level, e.g., the usual volume transformation software (filtering, symmetrization, down-sampling), graphical editors for on-screen design of tight masks and grayscale volume browsers. Many of these 
tools include bidimensional counterparts, allowing manipulation and annotation of micrographs. Below, we describe the manual alignment and subboxing tools.

\section{Manual alignment}

The dynamo_gallery tool allows picked particles to be visually inspected and their approximate orientation to be manually defined (for instance, in the case of elongated particles). This means that the automated refinement procedure subsequently carried out to obtain a more precise estimate for each particle to be restricted to a limited search range, facilitating the production of a starting average of roughly aligned particles. Even for sets containing tens or hundreds of thousands of particles, where the manual alignment of all particles is unrealistic, the ability to manually align a selected subset of particles to construct an initial average is a useful feature (Figure 7). It is also possible to estimate the resolution of the initial average by calculating the Fourier shell correlation (FSC) between independent averages generated from the two halves of the particles. This correlation does not involve any template-based alignment steps and therefore should not suffer from highresolution bias or overfitting due to alignment reference bias.

\section{Subboxing}

The dynamo_data_subboxing tools address the situation encountered when the structure averaged in an STA refinement contains several building elements. Averaging such a structure will not directly provide information about variances in the location and conformation of the building elements in the different particles of the data set. Instead, it approximately locates the elements inside each particle box of the data set. We call the use of the refinement parameters of the large structure to locate the individual positions and orientations of the building elements inside each original particle box subboxing. This applies, e.g., to particles that are ordered in a larger symmetry-related structure (such as in helical or 2D crystalline membrane arrangements). After aligning the larger structures (possibly by imposing the assumed symmetry operation), the subboxing tool automatizes the extraction of the asymmetrical subunits, and formats them to refine their alignments in an independent refinement experiment or to perform particle classification. A detailed example of an application can be found in (Gil-Carton et al., 2015), where an initial alignment of massive capsids of the Haloarcula hispanica icosahedral virus 2 (HHIV2) (Figure 8) was used to extract the twelve C5 vertices evident in each original subtomogram. Those vertices where then used as units for simultaneous alignment and classification by MRA in Dynamo. The procedure revealed that a spike was only present in a subset of 
vertices, and that the number and distribution of the vertices in the subset varied from virus to virus (Figure 9). This would not have been revealed by direct classification of the entire capsids: while individual vertices belong to one of two classes (spike on or off), the entire capsids belong to one of 4096 (i.e., $2^{\wedge} 12$ ) possible classes, depending on the combined states of their twelve vertices.

Generally, subboxes contain less signal per particle than the original subtomograms, and alignment should be performed with caution. The use of refinement tools will only improve the resolution if the SNR of the subtomograms is sufficient to improve the alignment that was obtained from the geometric constraints applied to the larger volume. Classification routines by PCA and K-means (Forster et al., 2008) do not require an alignment step and may be safely combined with subboxing.

Subboxing may be used to optimize computational efficiency, as shown for the determination of structure of the Yersinia enterocolitica injectisome, which is located between two bacterial membranes (Bharat et al., 2015; Kudryashev et al., 2013). The binned particles were manually aligned and refined by cross correlation. Next, the nonbinned particles were subboxed (without symmetry imposition) at the bacterial inner and the outer membranes, aligned independently, and then merged into the final structure. As the alignment of smaller particles is faster, the computation time was significantly reduced.

A bidimensional counterpart of the hereby described subboxing technique has already been proposed for Single Particle computations (Ilca et al., 2015), where density projections corresponding to a substructure of a previously aligned structure are extracted from the micrographs and treated as independent particles (there called subparticles) for further alignment and classification. Extraction of substructure projections requires the implementation of some policy to mitigate the fact that some subparticles will overlap with the projection of the rest of the structure. This problem is inherently solved in the context of subtomogram averaging, at the cost of typically attaining lower resolutions.

\section{Independent half-set refinement ("gold standard" processing).}

The Catalogue system leads the user all the way from tomogram sets to STA results. Thus, it also includes functionalities that interface and extend the previously available Dynamo library for the creation of alignment and classification projects. Besides more advanced scripting options, the GUI dcp presents the users simplified and expert pipelines for the design of simple or multi-reference alignment projects, connecting them with posterior 
PCA and Kmeans-based classification, and visualization of the results.

The simplified modus allows the user ( $\mathrm{Li}$ et al., 2016) to automatically perform the computations along the lines of a "gold standard" procedure. The "gold standard" method refers to an approach devised in single particle cryo-EM in order to avoid reference bias from causing correlations in reconstructions, thereby leading to artefactual reconstructions that suffer from overfitting, resolution loss, and at the same time, overestimation of resolution (Scheres and Chen, 2012). As overestimation of resolution may also occur in STA (Kudryashev et al., 2012), the dcp includes the "adaptive_bandpass" option for STA (Figure ), which divides subtomograms into two datasets and generates two initial tables by splitting an original table into even- and odd-numbered particle subpopulations. Initial references without high-resolution details should be used, and a very conservative low-pass filter should be specified (adaptive_initial_bandpass inside the area for advanced numerical parameters in $\mathrm{dcp}$ ). Two independent iterations are then run; at the end of the two iterations, two resulting structures are aligned by cross correlation, and the attained resolution is computed by dermining the FSC between them. This FSC profile is then used to define the resolution limitation of the reference for the next iteration, which is used as a cut-off value when computing the cross-correlation between reference and data particles.

\section{Conclusions}

We present a set of semi-automated particle picking tools for subtomogram processing, that are integrated in the Dynamo pipeline. These tools accelerate particle picking and at the same time provide initial orientations for the particles. This orientation information allows local searches for particle shifts and rotations to be performed, which speeds up processing and minimizes the amount of misaligned particles. Knowledge of initial orientations for all or a subset of the particles ensures that a reliable initial reference is generated for future alignment. Particle picking is incorporated into the Dynamo workflow by a database called Dynamo Catalogue, which can also deliver its output into other STA package. This database will enable users to approach complex problems in STA, particularly in membrane protein research.

Dynamo is freely available for download through the webpage www.dynamo-em.org. Installing Dynamo does not require the purchase of a license for Matlab or any other 
commercial software.

\section{Acknowledgements:}

We thank Nikolaus Stahlberg for support with beta-testing, Marcel Arheit, Julia Kowal, Juha Huiskonen, Alex Noble and Sai Li for useful discussions and user feedback, and Takashi Ishikawa, Bara Malkova, and Morgan Beeby for sharing tomographic data for visualization purposes. This work was in part supported by the Swiss Initiative for Systems Biology SystemsX.ch (RTD CINA) and by the Swiss National Science Foundation (NCCR Transcure and grant 205320_144427). 


\section{References}

\section{Reforences}

Amat, F., Comolli, L.R., Nomellini, J.F., Moussavi, F., Downing, K.H., Smit, J., Horowitz, M., 2010. Analysis of the intact surface layer of Caulobacter crescentus by cryo-electron tomography. J Bacteriol 192, 5855-5865.

Amenta, N., Bern, M., Eppstein, D., 1998. The crust and the beta-skeleton: combinatorial curve reconstruction. Graphical Models and Image Processing 60, 125-135.

Bartesaghi, A., Lecumberry, F., Sapiro, G., Subramaniam, S., 2012. Protein secondary structure determination by constrained single-particle cryo-electron tomography. Structure 20, 2003-2013.

Beck, M., Lucic, V., Forster, F., Baumeister, W., Medalia, 0., 2007. Snapshots of nuclear pore complexes in action captured by cryo-electron tomography. Nature 449, $611-615$.

Bharat, T.A., Russo, C.J., Lowe, J., Passmore, L.A., Scheres, S.H., 2015. Advances in SingleParticle Electron Gryomicroscopy Structure Determination applied to Subtomogram Averaging. Structure 23,1743-1753.

Bharat, TA., Davey, N.E., Ulbrich, P., Riches, J.D, de Marco, A., Rumlova, M., Sachse, C., Ruml, T., Briggs, J.A., 2012. Structure of the immature retroviral capsid at 8 A resolution by cryo-electron microscopy. Nature 487, 385-389.

Briggs, J.A., 2013. Structural biology in situ--the potential of subtomogram averaging. Gurrent opinion in structural biology 23, 261-267.

Castano-Diez, D., Kudryashev, M., Arheit, M., Stahlberg, H., 2012. Dynamo: a flexible, userfriendly development tool for subtomogram averaging of cryo-EM data in highperformance computing environments. J Struct Biol 178, 139-151.

Dudkina, K.V., Kudryashev, M., Stahlberg, H., Boekema, E.J, 2011. Interaction of complexes I, III, and IV within the bovine respirasome by single particle cryoelectron tomography. Proc. Natl. Acad. Sci. U. S. A. 108, 15196-15200.

Faini, M., Beck, R., Wieland, F.T., Briggs, J.A., 2013. Vesicle coats: structure, function, and general principles of assembly. Trends Cell Biol 23, 279-288.

Forster, F., Pruggnaller, S., Seybert, A., Frangakis, A.S., 2008. Classification of cryo-electron sub-tomograms using constrained correlation. J Struct Biol 161, 276-286.

Forster, F., Medalia, O., Zauberman, N., Baumeister, W., Fass, D., 2005. Retrovirus envelope protein complex structure in situ studied by cryo-electron tomography. Proc. Natl. Acad. Sci. U. S. A. 102, $4729-4734$.

Frangakis, A.S., Bohm, J., Forster, F., Nickell, S., Nicastro, D., Typke, D., Hegerl, R., Baumeister, W., 2002. Identification of macromolecular complexes in cryoelectron tomograms of phantom cells. Proc Natl Acad Sci U S A 99, 14153-14158.

Galaz-Montoya, J.G., Flanagan, J., Schmid, M.F., Ludtke, S.., 2015. Single particle tomography in EMAN2. J Struct Biol 190, 279-290.

Gil-Garton, D., Jaakkola, S.T., Gharro, D., Peralta, B., Gastano-Diez, D., Oksanen, H.M., Bamford, D.H., Abrescia, N.G., 2015. Insight into the Assembly of Viruses with Vertical Single beta-barrel Major Capsid Proteins. Structure 23, 1866-1877.

Harapin, J., Eibauer, M., Medalia, 0., 2013. Structural analysis of supramolecular assemblies by cryo-electron tomography. Structure 21, 1522-1530. 
Hrabe, T., Chen, Y., Pfeffer, S., Euellar, L.K., Mangold, A.V., Forster, F., 2012. PyTom: a pythonbased toolbox for localization of macromolecules in cryo-electron tomograms and subtomogram analysis. J. Struct. Biol. 178, 177-188.

Huiskonen, J.T., Hepojoki, J., Laurinmaki, P., Vaheri, A., Lankinen, H., Butcher, S.J., Grunewald, K., 2010. Electron cryotomography of Tula hantavirus suggests a unique assembly paradigm for enveloped viruses. J Virol 84, 4889-4897.

Ilca, S.L., Kotecha, A., Sun, X., Poranen, M.M., Stuart, D.I., Huiskonen, J.T., 2015. Localized reconstruction of subunits from electron cryomicroscopy images of macromolecular complexes. Nat Commun 6, 8843.

Kudryashev, M., Eastano-Diez, D., Stahlberg, h., 2012. Limiting factors in single particle cryo electron tomography. Comput Struct Biotechnol J 1, 201207002.

Kudryashev, M., Stenta, M., Schmelz, S., Amstutz, M., Wiesand, U., Gastano-Diez, D., Degiacomi, M.T., Munnich, S., Bleck, G.K., Kowal, J., Diepold, A., Heinz, D.W., Dal Peraro, M., Eornelis, G.R., Stahlberg, H., 2013. In situ structural analysis of the Yersinia enterocolitica injectisome. Elife 2, e00792.

Kunz, M., Frangakis, A.S., 2014. Super-sampling SART with ordered subsets. J Struct Biol 188, 107-115.

Li, S., Sun, Z., Pryce, R., ParSy, M.L., Fehling, S.K., Schlie, K., Siehert, G.A., Garten, W., Bowden, T.A., Strecker, T., Huiskonen, J.T., 2016. Acidic pH-Induced Conformations and LAMP1 Binding of the Lassa Virus Glycoprotein Spike. PLoS Pathog 12, e1005418.

Lucic, V., Rigort, A., Baumeister, W., 2013. Gryo-electron tomography: the challenge of doing structural biology in situ. J Gell Biol 202, 407-419.

Maurer, U.E., Zeev-Ben-Mordehai, T., Pandurangan, A.P., Eairns, T.M., Hannah, B.P., Whitheck, J.G., Eisenberg, R.J., Gohen, G.H., Topf, M., Huiskonen, J.T., Grunewald, K., 2013. The structure of herpesvirus fusion glycoprotein B-bilayer complex reveals the protein-membrane and lateral protein-protein interaction. Structure 21, 1396-1405.

Nicastro, D., Schwartz, G., Pierson, J., Gaudette, R., Porter, M.E., McIntosh, J.R., 2006. The molecular architecture of axonemes revealed by cryoelectron tomography. Science 313, 944-948.

Peralta, B., Gil-Garton, D., Gastano-Diez, D., Bertin, A., Boulogne, C., Oksanen, H.M., Bamford, D.h., Abrescia, K.G., 2013. Mechanism of membranous tunnelling nanotube formation in viral genome delivery. PLoS Biol 11, e1001667.

Pfeffer, S., Brandt, F., Hrabe, I., Lang, S., Eibauer, M., Zimmermann, R., Forster, F., 2012. Structure and 3D arrangement of endoplasmic reticulum membrane-associated ribosomes. Structure 20, 1508-1518.

Pigino, G., Bui, K.H., Maheshwari, A., Lupetti, P., Diener, D., Ishikawa, T., 2011. Gryoelectron tomography of radial spokes in cilia and flagella. J Cell Biol 195, 673-687.

Scheres, S.H., Chen, S., 2012. Prevention of overfitting in cryo-EM structure determination. Nat Methods 9, 853-854.

Schur, F.K., Hagen, W.J., de Marco, A., Briggs, J.A., 2013. Determination of protein structure at 8.5A resolution using cryo-electron tomography and sub-tomogram averaging. J. Struct. Biol. 184, 394-400.

Schur, F.K., Hagen, W.J., Rumlova, M., Ruml, T., Muller, B., Krausslich, H.G., Briggs, J.A., 2015. Structure of the immature HIV-1 capsid in intact virus particles at 8.8 A resolution. Nature 511, 505-508. 


\section{Tables}

Table 1. Summary of the options for particle picking in various geometries

\begin{tabular}{|c|c|c|c|}
\hline $\begin{array}{c}\text { Dynamo } \\
\text { Model Type }\end{array}$ & User Intervention & Initial Particle Positions and Orientation & Specific tools: \\
\hline General & - Clicking centers of particles. & $\begin{array}{l}\text { - Positions: directly from clicking } \\
\text { - Orientations: initialized as defaults (angles set to } \\
\text { zero) } \\
\text { - Originating from a reference model: parallel to a } \\
\text { filament or outwards from a membrane. }\end{array}$ & $\begin{array}{l}\text { - Automatic coordination of intensity views } \\
\text { from different orthogonal planes to expose } \\
(\mathrm{X}, \mathrm{Y}) \text { location and Z-depth of an isolated } \\
\text { particle (dtomoview). }\end{array}$ \\
\hline $\begin{array}{l}\text { Surface } \\
\text { (Membrane- } \\
\text { ByLevels) }\end{array}$ & $\begin{array}{l}\text { - Clicking points coarsely defining a } \\
\text { membrane or an arbitrary surface in } \\
\text { 3D slices }\end{array}$ & $\begin{array}{l}\text { - Positions: sampled on the smoothly approximated } \\
\text { surface defined by clicked points } \\
\text { - Orientations: defined as the normal to the } \\
\text { membrane plane }\end{array}$ & $\begin{array}{l}\text { - Controls for the creation of smooth re- } \\
\text { interpolated membrane planes (2D } \\
\text { meshes). }\end{array}$ \\
\hline 3D Crystal & $\begin{array}{l}\text { - Clicking points to define the } \\
\text { boundaries of the crystal in 3D, as } \\
\text { done for MembraneByLevels } \\
\text { - Defining a general orientation. }\end{array}$ & $\begin{array}{l}\text { - Positions: regularly sampled within the 3D crystal } \\
\text { boundaries in user-defined spacings } \\
\text { - Orientations: initialized as defaults (angles set to } \\
\text { zero) or specified by the user as a single vector. }\end{array}$ & - None \\
\hline
\end{tabular}




\section{Captions}

Figure 1. The Catalogue database. Sketch of the STA workflow driven by Dynamo Catalogue. The raw data delivered by the microscope are several tilt series of projections. A reconstruction is performed for each series (top left). The resulting tomograms are then analyzed within Dynamo Catalogue through manual or semiautomatic methods, using models that ultimately define the approximate positions and orientations of particles. The Catalogue database records all the parameters, allowing the particles to be extracted and formatted adequately for subtomogram averaging in Dynamo or other packages whenever required.

Figure 2. Concurrent viewing of isolated particles, demonstrated using a tomogram containing several copies of the bacteriophage PRD1 (as used in Peralta et al., 2013). (A) displays the projection along the direction of the beam, which is $\mathrm{Z}$ by convention. (B) is a closer view on this projection, where the user clicks on screen the approximate center of a projected phage. This is equivalent to selecting a pair of $\mathrm{X}$ and $\mathrm{Y}$ coordinates, represented by a cross with a segment in magenta that fixes coordinate $\mathrm{X}$, and a segment in green that fixes coordinate Y. Upon this click, Dynamo would show the user the plots (C) and (D), showing $\mathrm{Z}$ sections of the tomogram around the just clicked $\mathrm{X}$ and $\mathrm{Y}$. (C) is an $\mathrm{YZ}$ section, where the user can click a point (represented by the blue sphere) to select an YZ coordinate. The $\mathrm{X}$ coordinate is implicit by the section itself, and the blue point is thus completely defined in 3D. (D) shows the XZ section. The user can use (C) or (D) to define the blue point, depending on which one offers better visibility. (E) depicts the geometric relationship between (C), (D), (B) and the point defined by the user (in blue).

Figure 3. Filament model, demonstrated using an experimental dataset of microtubules. (A) X-Y section of a tomogram showing a bundle of bent filaments. (B) An editor allowing the display of selected cross sections orthogonal to the approximate path of a filament facilitates the identification of individual paths, revealing complex geometric configurations. (C) Filament models can be directly pipelined into an alignment project. (D) Result of an automatic multi-reference STA project, applied to the shown dataset, revealing different numbers of protofilaments in each tubular filament.

Figure 4. Membrane model, here demonstrated with a data set recorded to investigate a bacterial membrane in C. Crescentius. A pipeline for the extraction of particles from a membrane context. The red framed insets between the plots are the icons in the Dynamo interface that an user would click to launch the operators that implement the corresponding pipeline step. (A) Tomogram browsers allow the manual selection of landmarks along a membrane. These are initially fed into the membrane model as a cloud of points. (B) A click on the spline operator associated with the 
model identifies points that belong to same Z-level, creating an uniform distribution along the shape of the membrane, i.e. the grey points in $(\mathrm{C})$. The triangulation operator uses this regular distribution of points to generate a coarse mesh (D) that can be refined by subdivision (E) to produce a smooth surface that models the area of interest of the physical membrane. (F) On this surface, further tools define regular distributions of spatial positions, each furnished with an orientation $(G)$ normal to the membrane. $(\mathrm{H})$ This allows subtomograms to be extracted along the defined positions. Each step is stored in the catalogue for joint processing with other models defined in the tomogram set. It is also possible to directly sum the contents of a single model to obtain a 3D average (I) and to define a set of particles $(\mathrm{J})$, extract them and run an alignment project.

Figure 5. Model combinations, demonstrated using a dynein-decorated microtubule dataset. Dynein particles appear as irregular decorations on microtubules and require the application of a combined model. (A) Several orthoslices are needed to define the path of the filament (black points). (B) An oblique slice matching the natural path of a filament shows dynein particles (inside the blue circles) at the expected distance from the microtubule. (C) The dynein particles corresponding to a single filament visualized as colored points in 3D. The metadata of the particle registers its orientation relative to its reference microtubule. Each red-framed box in the montage in (D) depicts projections of subtomograms cropped on tomogram areas locations identified as dynein decorations. Before projection, each particle was rotated according to its position relative to its reference microtubule, in this case using the convention of $\mathrm{Z}$ being the direction of the microtubule and $\mathrm{Y}$ the direction of the shortest line between particle and microtubule. The overlaid 3D density is the average subtomogram (without refinement) of several hundreds of such particles, showing the coherence of the imparted orientations. Tomogram Courtesy of Drs. Takashi Ishikawa and Barbora Malkova (PSI, Villigen, Switzerland).

Figure 6. Semiautomatic procedures, demonstrated using liposomes embedding 5ht3 neuroreceptors. (A) $\mathrm{Z}$ projection of a tomogram. Blue circles are projections of full liposomes (for simplicity, not all visible projections are marked). A click on the center of a projected liposome generates an auxiliary image (shown adjacent to the respective circle) that allows the user to approximately identify the Z-coordinate of the liposome center. (B) The user-defined center is used as a seed to locate the vesicle by template matching against spheres of different radii. The computed center and radius are then used to instantiate a vesicle object, which controls the creation of particle orientation regularly distributed on its surface. The orientations of these particles are shown as blue lines normal to the modeled surface.

Figure 7. Manual orientation, demonstrated using a dataset recorded to study type-three secretion system of Yersinia enterocolitica in situ (Kudryashev et al., 2013). (A) Slice from a tomogram of a Y. enterocolitica bacterium with type 3 secretion injectisomes (red circles). (B) On-screen depiction of subtomogram particle projections allows an original orientation to be assigned to each by two 
mouse-clicks per subtomogram: "center" and "north". Demonstrated here for 20 projections. (C) Average of the 10 particles selected from B (marked blue). This average provides an initial template for further refinement.

Figure 8. Loss of local features by averaging heterogenous structures. (A) is a $\mathrm{Z}$ section of a tomogram containing several copies of a virus, a closer view of two of them is rendered on (B). The yellow boxes represent the three dimensional extent of the subtomograms that will be extracted from the full data set. In (C), each box represents the projection along $\mathrm{Z}$ of one different subtomogram (four subtomograms were arbitrarily chosen), and (D) represents the $\mathrm{Z}$ projection of the same subtomograms after alignment. The orange boxes represent spike-like densities that can be spotted on some viruses. These densities appear on symmetrically related sites, but on each virus they show in different numbers and in different locations. As a result, subtomogram averaging of whole viruses results in the density shown in (E), where spikes do not show in the final average.

Figure 9. Subboxing analysis on the data shown in Figure 8. (A) Subtomogram averaging of whole viruses provides an alignment table with positions and angles of each phage in its tomogram. (B) The position of a penton in the average (in a magenta subbox) is symmetrically related to the other eleven pentons (each one in an orange subbox). (C) Applying the symmetry operation sketched in (B) on the alignment table represented in (A) results in a table that describes separately positions and orientations (represented in orange) of all the pentons in the tomograms. This allows to extract them and create a new data set where each subtomogram is the subbox around a single penton. (D) depicts the result of this extraction. Each orange box is a projection of a different subtomogram. The vertical direction corresponds to the $\mathrm{C} 5$ symmetry axis. The red boxes show individual pentons that appear to have an spike attached. (E) shows the result of aligning and classifying the data set of individual pentons. Two different classes arise upon use of Multireference Analysis, showing pentons with and without a spike (in red and cyan respectively). The class membership of each penton can be used to reconstuct the architecture of each individual virus, as depicted in (F).

Figure 10. Schematic representation of the gold-standard alignment method implemented in Dynamo. The dataset is split into two sub-sets with even- and odd-numbered subtomograms. These are independently aligned and averaged. Calculation of the FSC between the two averages is used to determine the resolution of the merged average reconstruction, which can then be filtered with the FSC resolution profile to generate a suitable reference for further alignment iteration cycles. 
Tomograms

Models
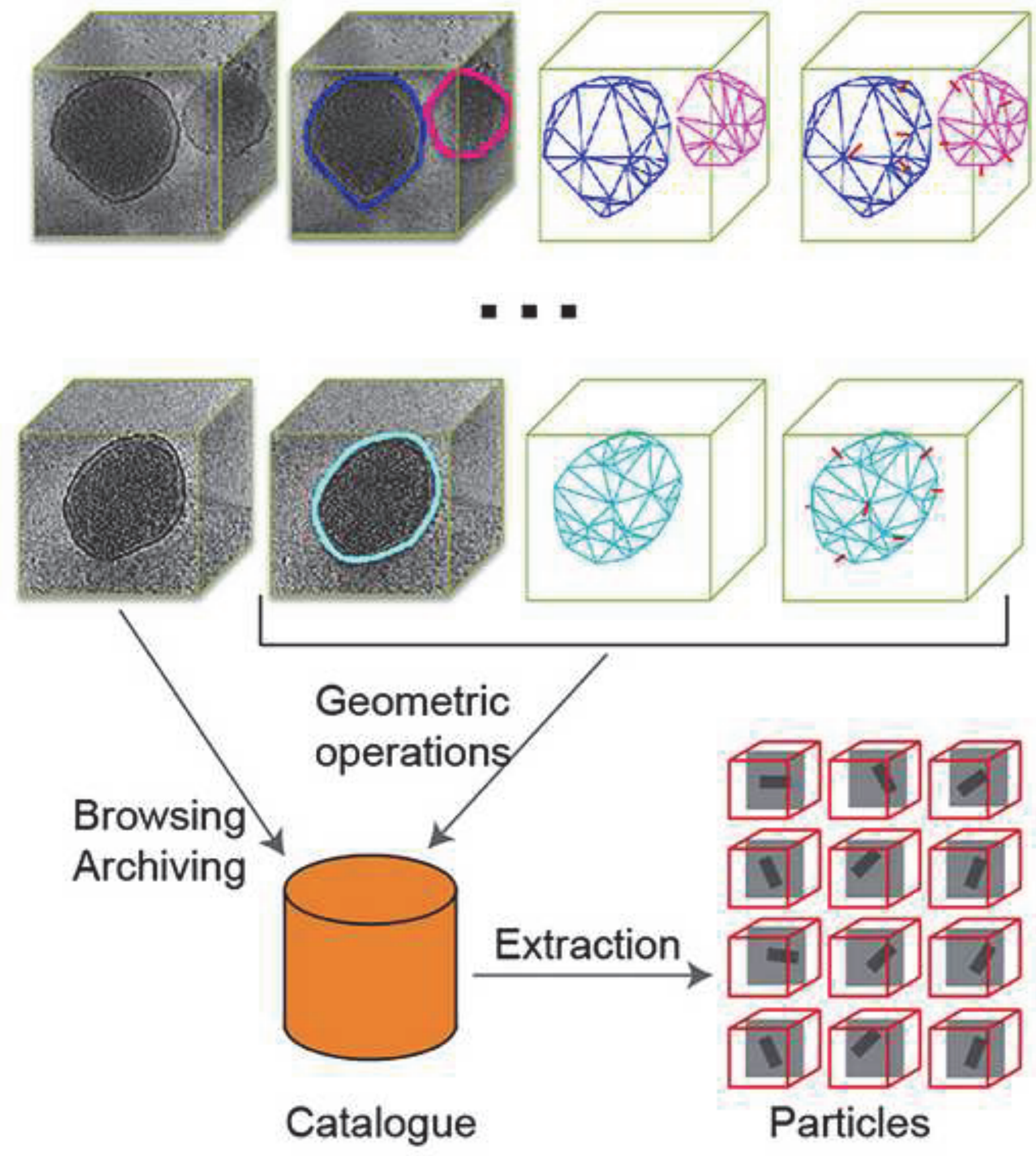


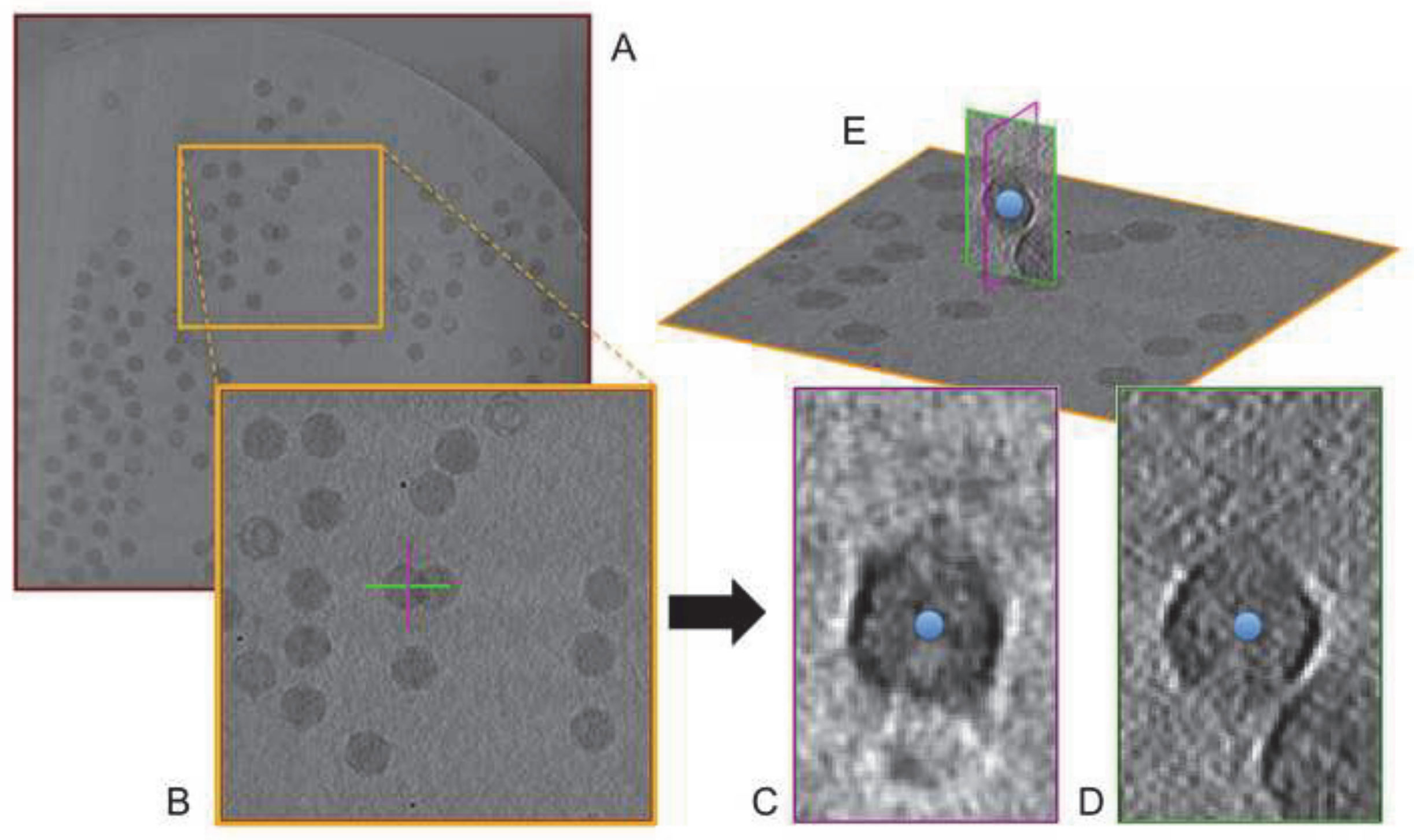




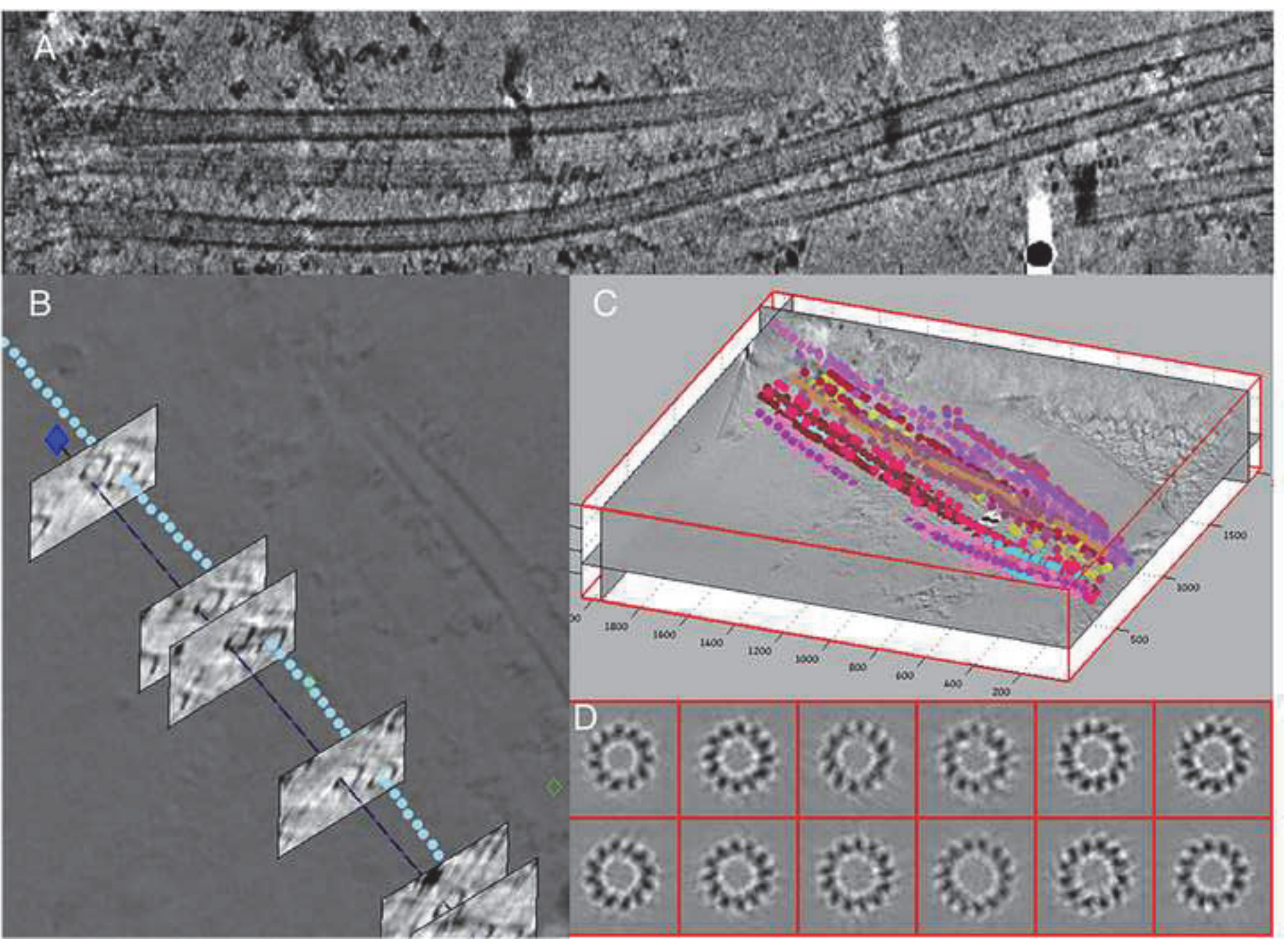



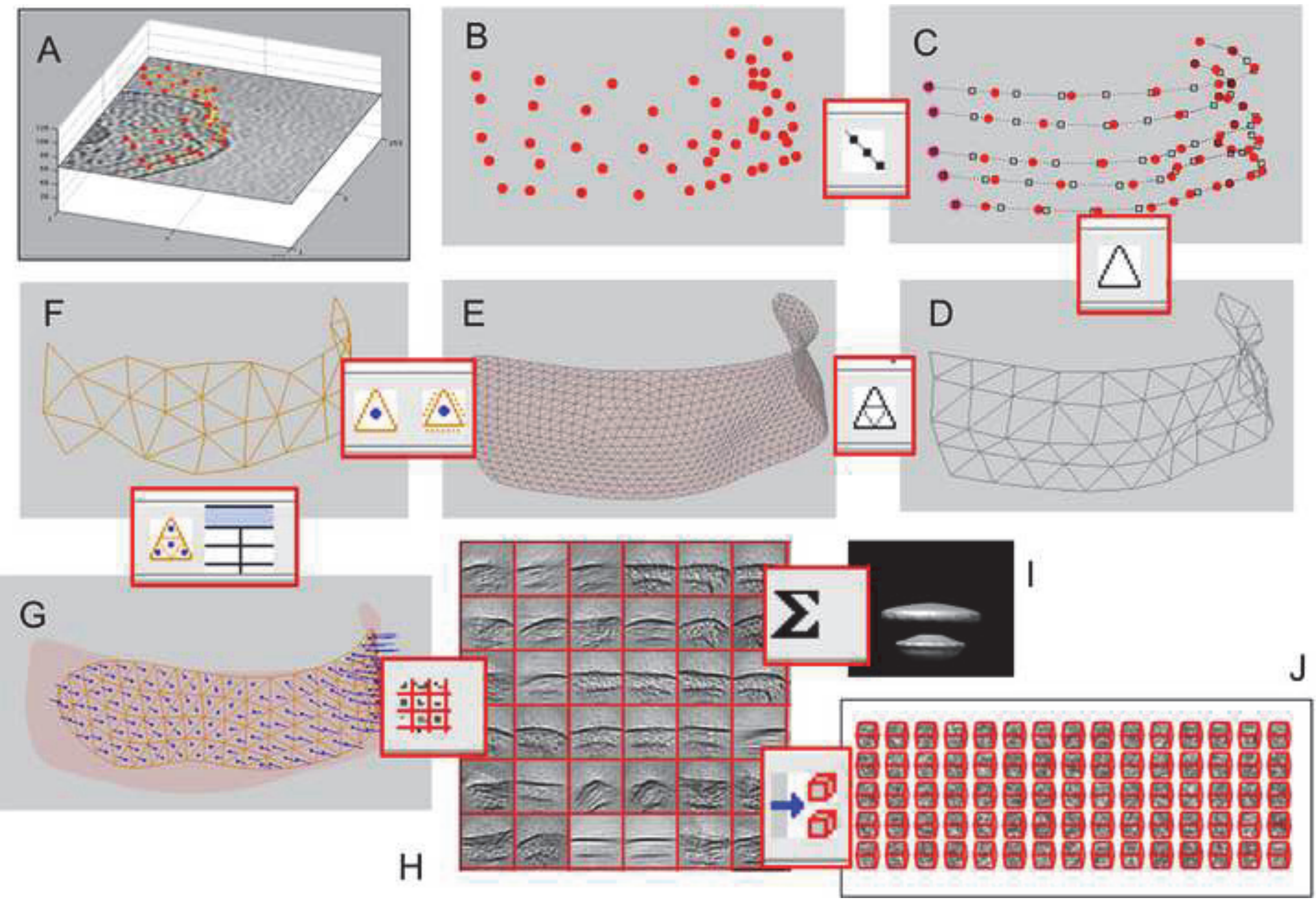


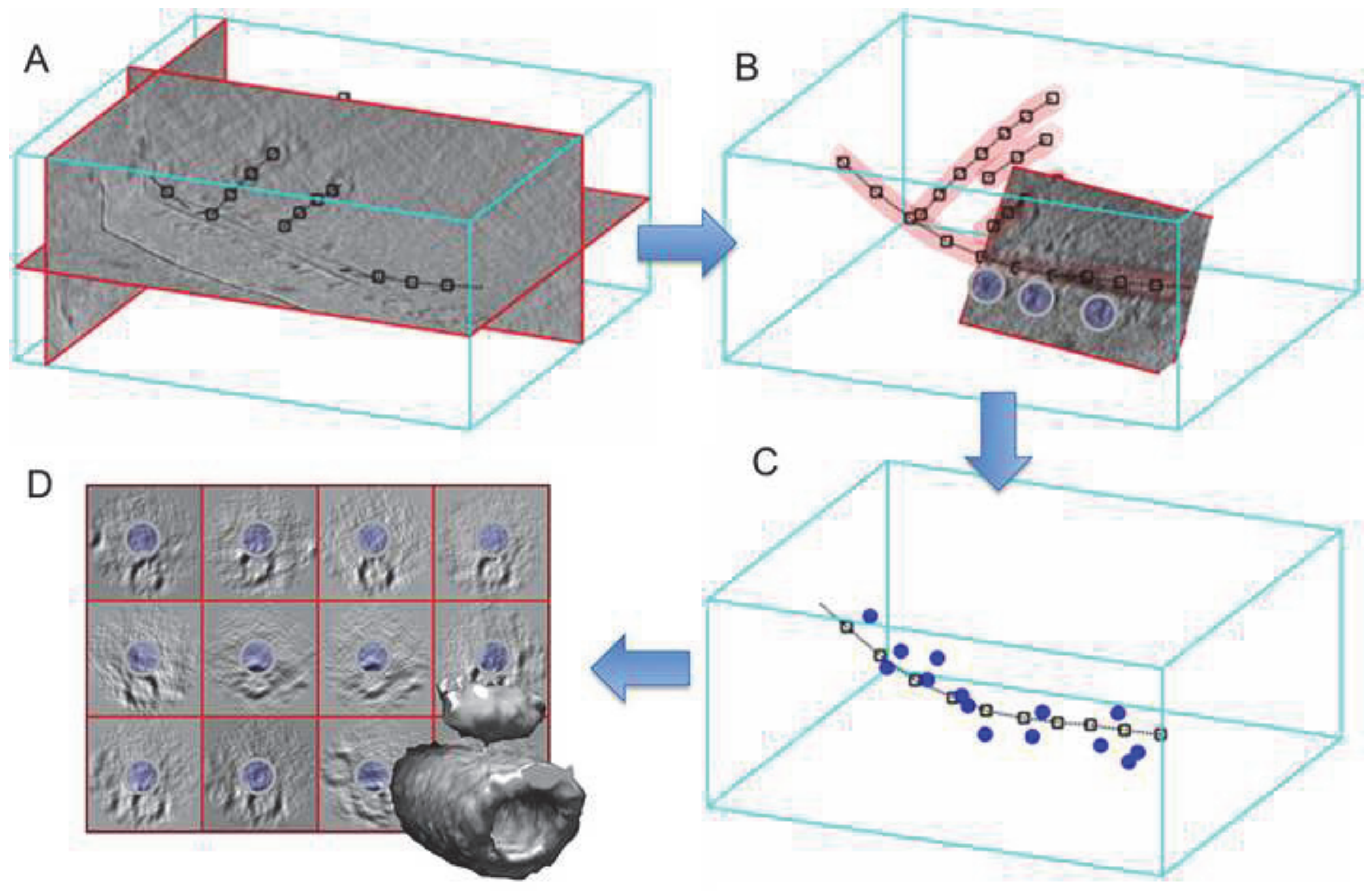




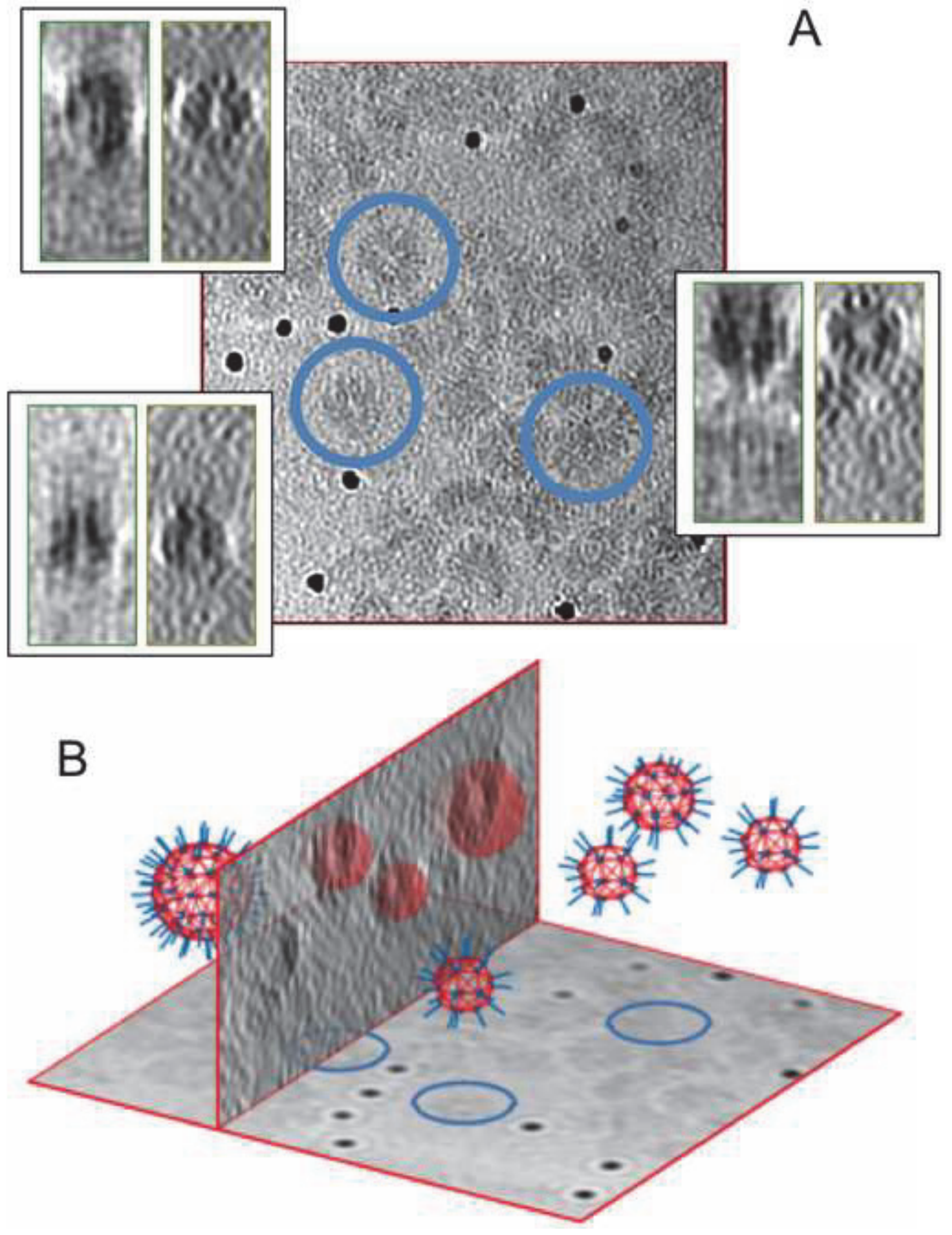




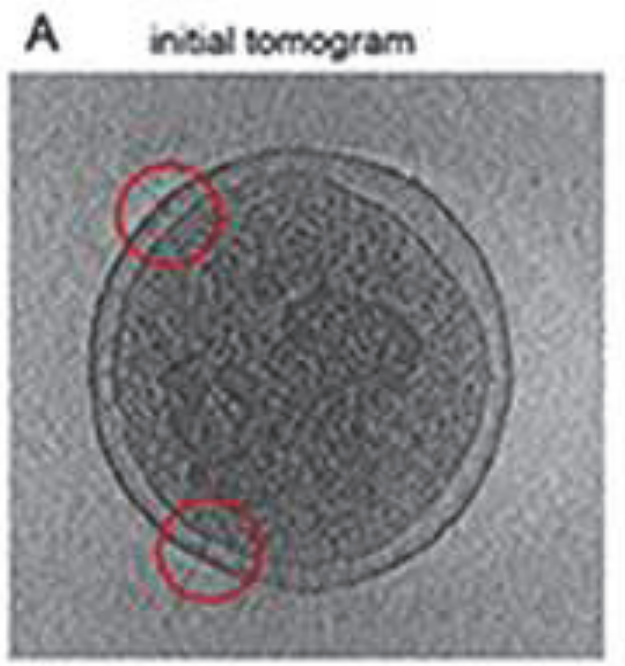

B
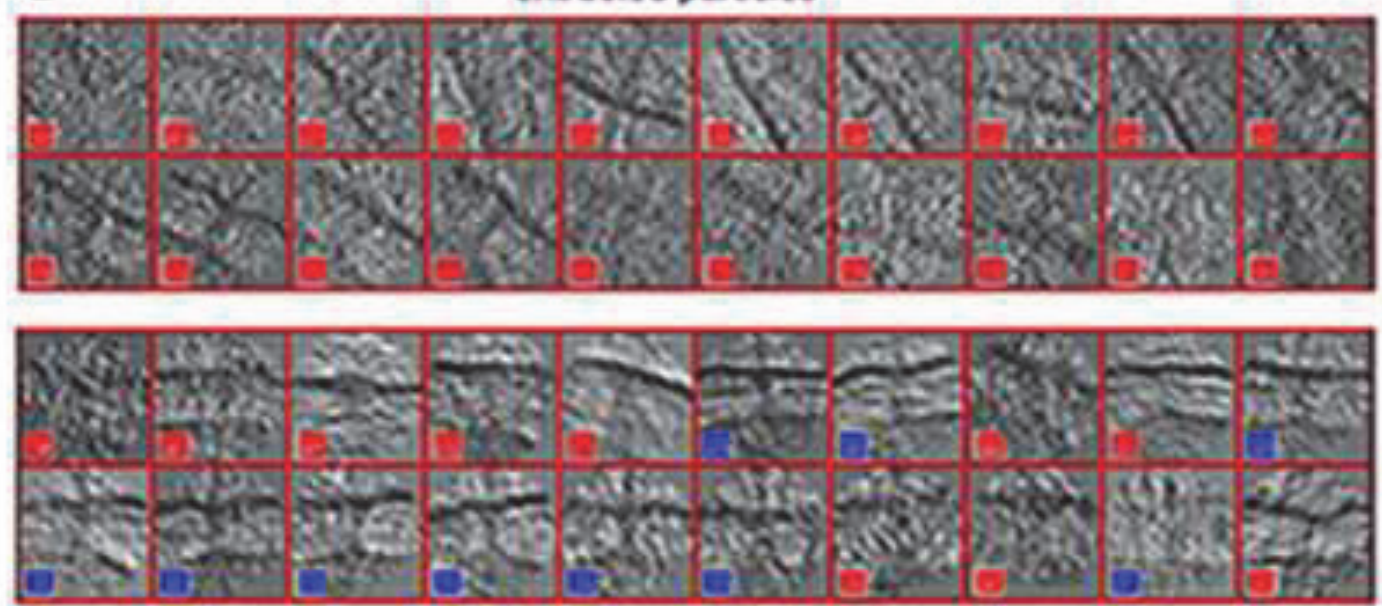

manually aloned particles, blue labels indicate selection
Caverape of 21 particles
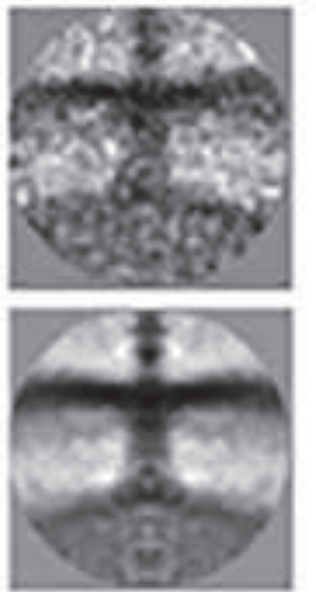

symmetrized average 


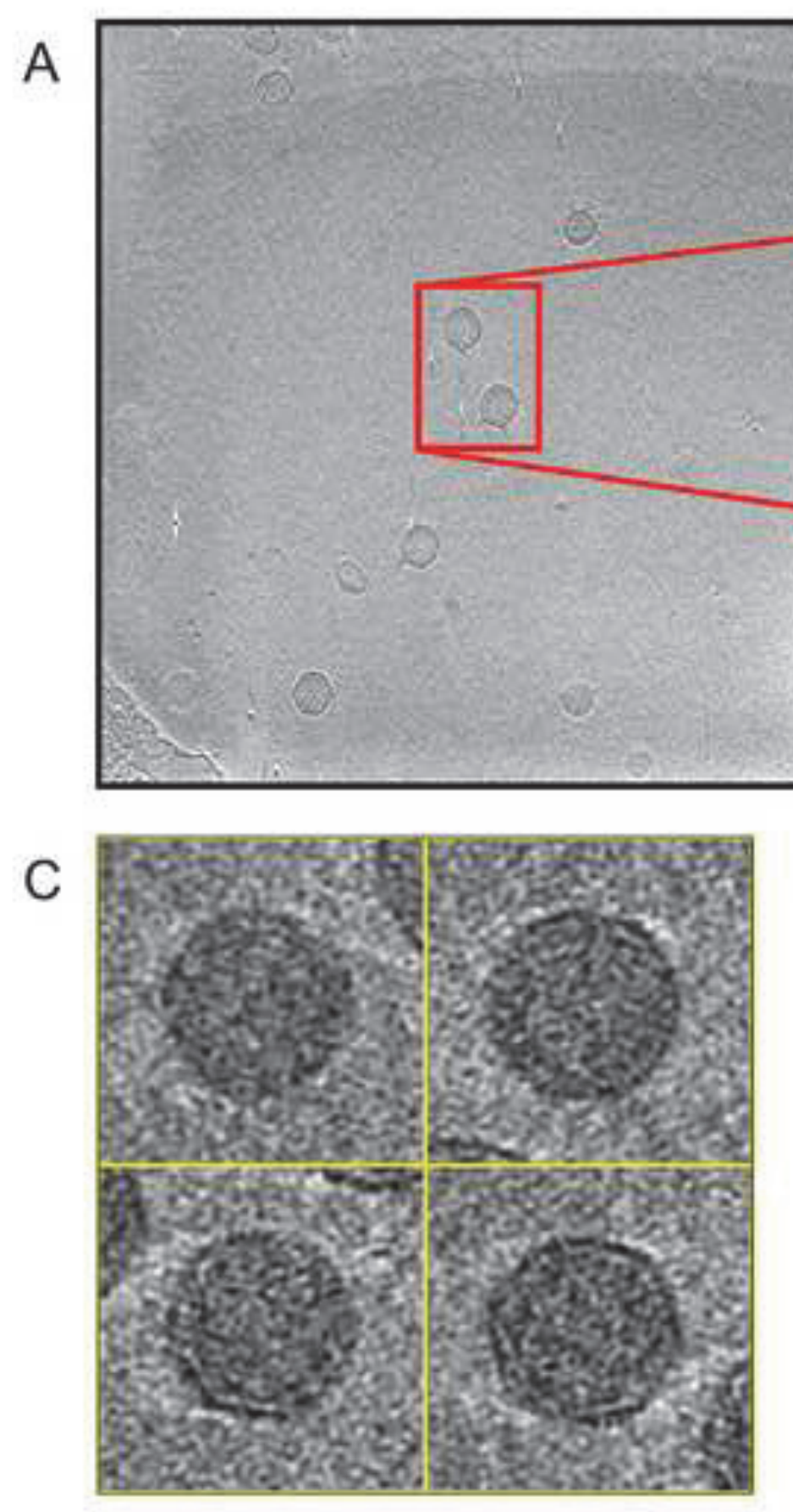

B

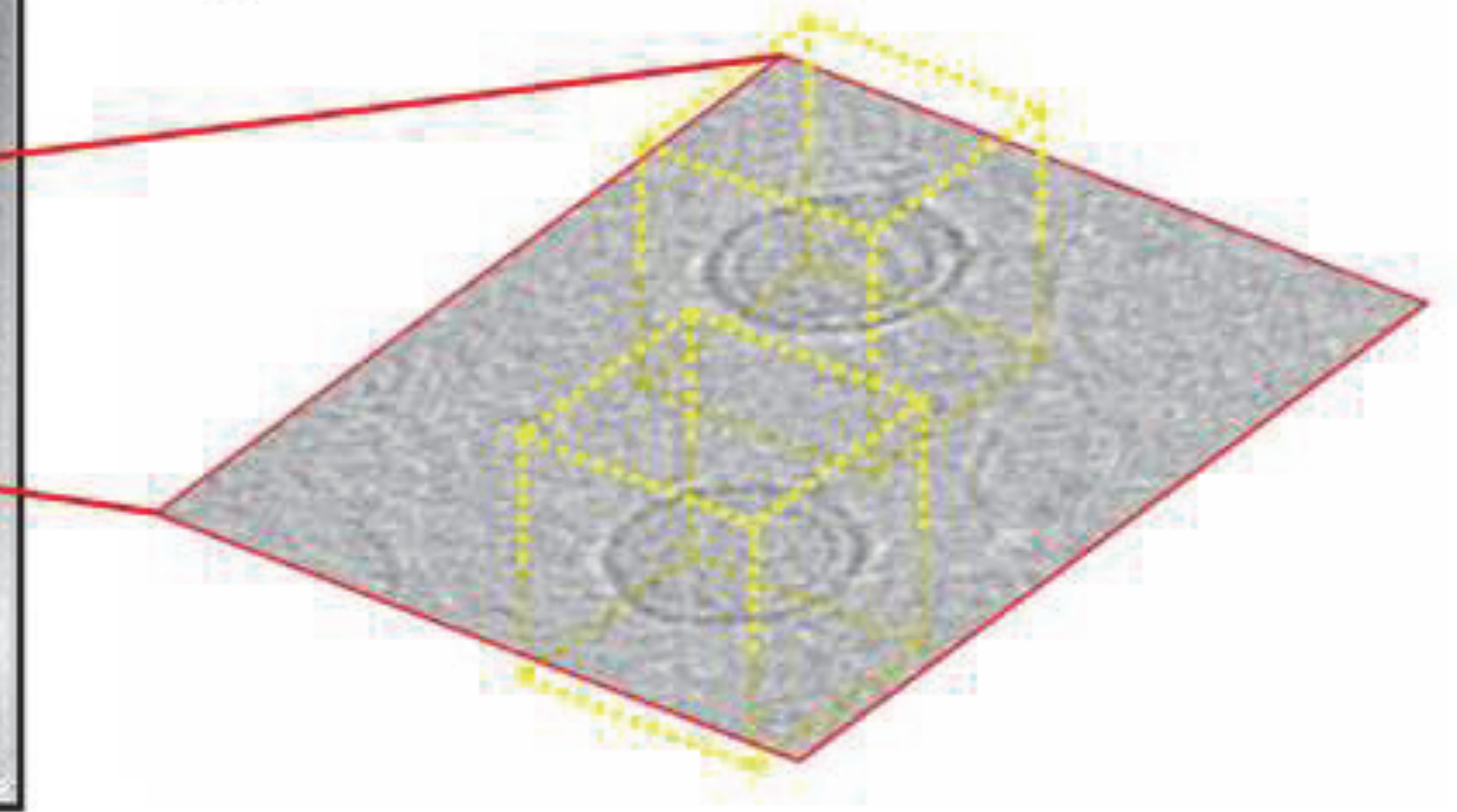

D
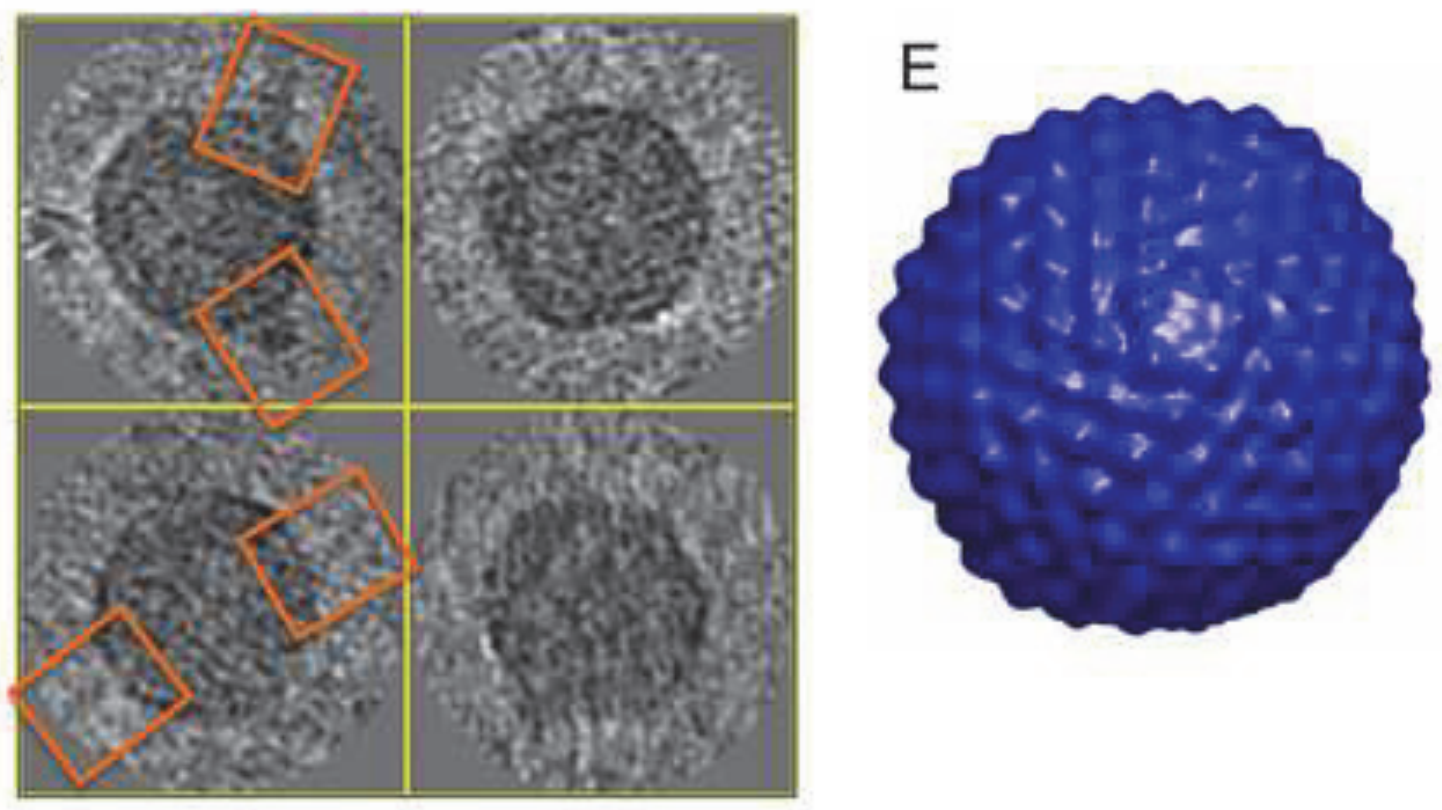


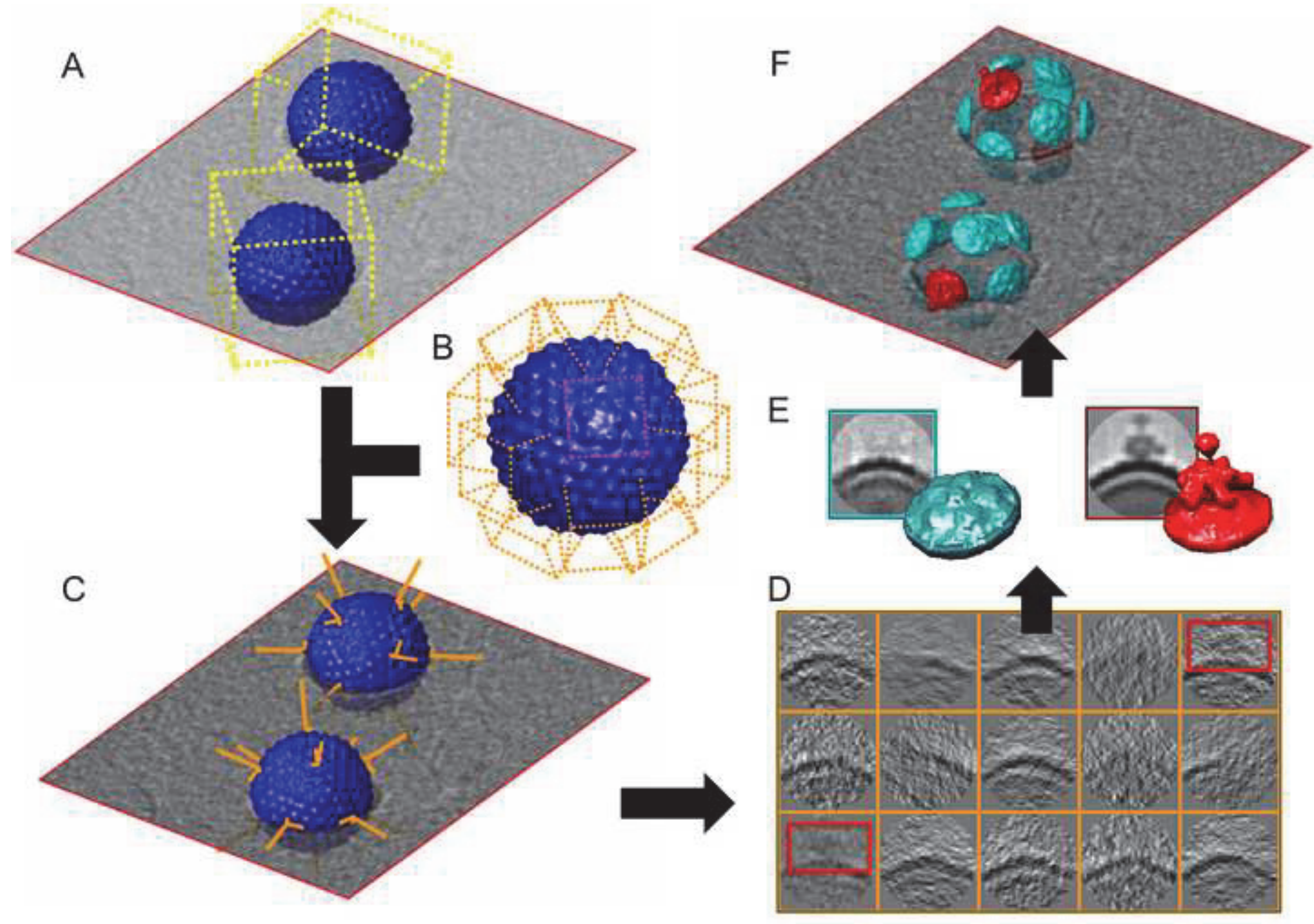




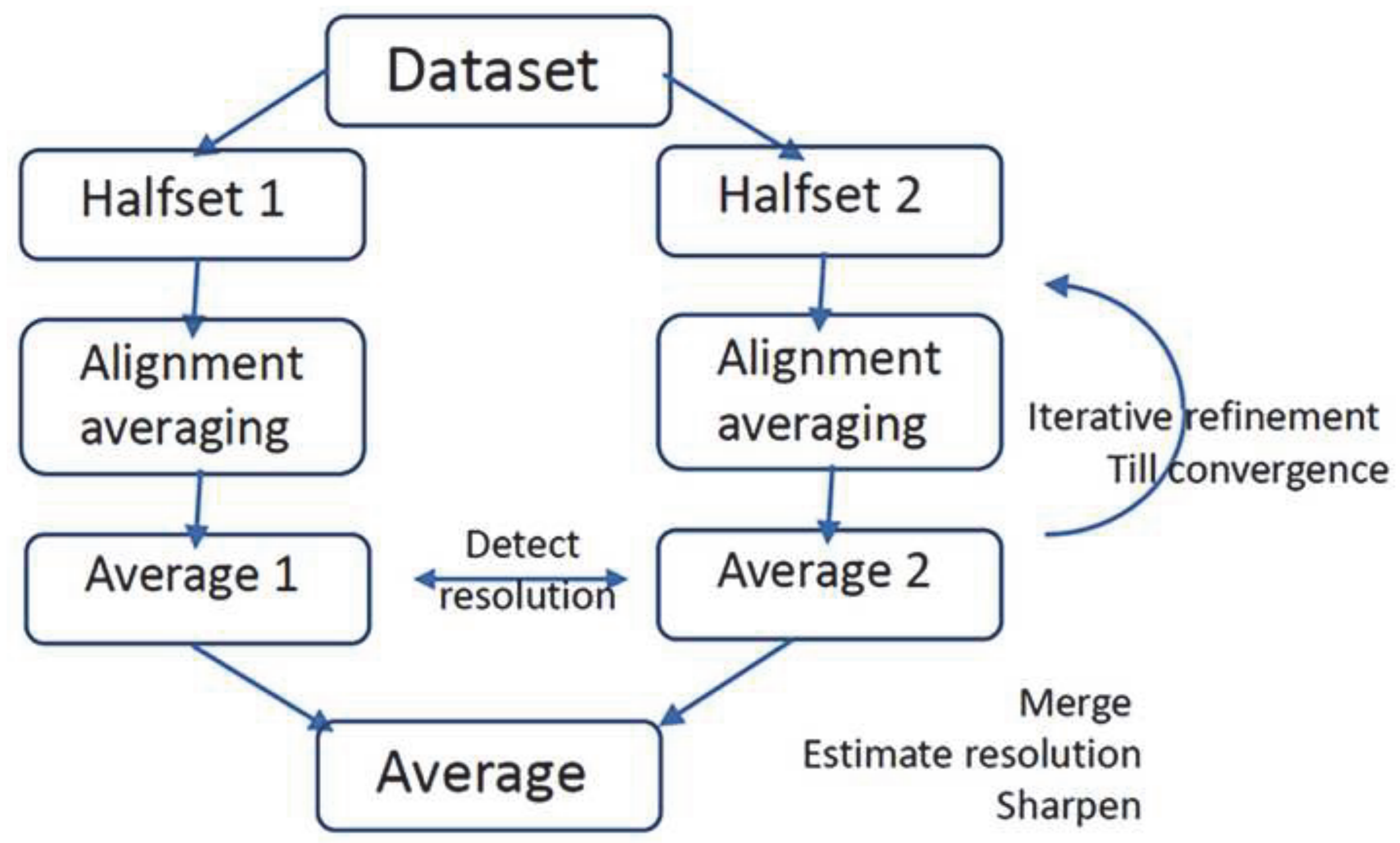

